\title{
Estrogenic Activity of Branched 4-Nonylphenol Isomers Examined by Yeast Two-Hybrid Assay
}

\author{
Hirotaka Shioji, ${ }^{a}$ Shinji Tsunoi, ${ }^{*, a}$ Yosuke Kobayashi, ${ }^{a}$ Tatsushi Shigemori, ${ }^{b}$ Michihiko Ike, ${ }^{b}$ \\ Masanori Fujita, ${ }^{b, 1}$ Yoko Miyaji, ${ }^{c}$ and Minoru Tanaka ${ }^{a}$
}

${ }^{a}$ Research Center for Environmental Preservation, Osaka University, 2-4 Yamada-oka, Suita, Osaka 565-0871, Japan, ${ }^{b}$ Department of Environmental Engineering, Graduate School of Engineering, and ${ }^{c}$ Analytical Center, Faculty of Engineering, Osaka University, 2-1 Yamada-oka, Suita, Osaka 565-0871, Japan

(Received October 29, 2005; Accepted December 21, 2005; Published online December 26, 2005)

\begin{abstract}
Various branched isomers of 4-nonylphenol (NP) and, in addition, the other 4-alkylphenols (APs) were synthesized and their estrogenic activities were assessed using the yeast two-hybrid system. We investigated the relationships between the structure of the nonyl group of NP isomers and their estrogenic activities based on the following five factors: the length of the main alkyl chain, the degree of branching on the $\alpha$-carbon, the degree of bulkiness, the position of the branch, and the cyclic structure in the nonyl group. An appropriate length of the main alkyl chain was essential for the estrogenic activity. A small effect of the branching on the $\alpha$-carbon was observed. The importance of the bulkiness and position of the branch in the nonyl group was suggested from the results of the synthesized NP isomers possessing the high estrogenic activities. The bulkiness on the $\beta$-carbon was the most important factor for the high estrogenic activity. The investigation of the cyclic structure also indicated the significance of the bulkiness around the $\beta$-carbon. The bulkiness on the $\gamma$-carbon was also suggested to be an important factor. The metabolic effect of the synthesized APs on the estrogenic activity was also examined using a liver S9. The estrogenic activities of the selected APs were either reduced or lost. In addition, GC-MS analyses of the commercial NP and synthesized NP isomers revealed that the nine synthesized NP isomers were included in the commercial NP.
\end{abstract}

Key words — alkylphenol, estrogenic activity, nonylphenol, S9, yeast two-hybrid assay

\section{INTRODUCTION}

Endocrine disruption by artificial chemicals has raised worldwide concern because of the suspicion of the bad effect on animals and human health. ${ }^{1-3)}$ Some alkylphenols (APs), e.g., 4-octylphenol and 4-nonylphenol (NP), which are the breakdown products of non-ionic surfactants, are suspected to be endocrine disruptors. ${ }^{4,5)}$

The process of the endocrine disruption with APs is based on their binding to the ligand binding domain in the estrogen receptor. In other words, the APs mimic the action of $17 \beta$-estradiol (E2). In 1997 , Brzozowski et al. $^{6}$ ) reported the X-ray study of the crystal structure of the ligand binding domain of the

${ }^{1}$ Present address: Kochi National College of Technology, 200-1 Mononobe, Nangoku, Kochi 783-8508, Japan

*To whom correspondence should be addressed: Research Center for Environmental Preservation, Osaka University, 2-4 Yamada-oka, Suita, Osaka 565-0871, Japan. Tel.: +81-6-68798977; Fax: +81-6-6879-8978; E-mail: tsunoi@epc.osaka-u.ac.jp estrogen receptor $\alpha(\mathrm{ER} \alpha)$ in the complex with $\mathrm{E} 2$. The 3-hydroxyl group in E2 makes the hydrogenbonding interactions with the carboxyl group in Glu 353 and guanidinium group in $\operatorname{Arg} 394$, and the skeleton of E2 makes the van der Waals interaction with the hydrophobic site in the ligand binding domain. The 17 $\beta$-hydroxyl group in E2 also makes the hydrogen-bonding interaction with His 524. Because the hydrogen-bonding interaction of the $17 \beta$-hydroxyl group with His 524 is more flexible than that of the 3-hydroxyl group with Glu 353 and Arg 394, in addition, the size of the binding pocket in the ligand binding domain $\left(450 \AA^{3}\right)$ is almost two times larger than the volume of E2 $\left(245 \AA^{3}\right)$, thus the endocrine disruptors like the APs can be accepted into the ligand binding domain.

The estrogenic activities of several alkylphenolic compounds have been examined by in-vitro and invivo assays. ${ }^{7-11)}$ It was reported that the alkyl group on the para-position was the vital factor for the estrogenic activity. ${ }^{12)}$ Moreover, Routledge and Sumpter ${ }^{13)}$ recognized that the length of the alkyl 
group and tert-branch on the $\alpha$-carbon were related to the high estrogenic activity. The recombinant yeast assay results from Schultz et al. ${ }^{14)}$ indicated that the increased size and symmetry in the alkyl group enhanced the estrogenic activity.

Among the APs, NP has been frequently detected in environmental situation such as river water and fish as a mixture of branched isomers. ${ }^{15,16)}$ Gas chromatographic analysis indicated that more than 21 isomers were included in commercial NP. ${ }^{17)}$ Some NP isomers were fractionated by HPLC and their estrogenic activities were measured by recombinant yeast screen assay. ${ }^{18)}$ It was reported that the individual isomeric fractions of the commercial NP had different estrogenic activities. Therefore, for the exact evaluation of the estrogenicity of NP, the information on the estrogenic activities of the respective NP isomers is required. ${ }^{19)}$ In addition, the investigation of the relationships between the estrogenic activity and structure of NP is suggested to be essential. Because the complete separation of the commercial NP to pure NP isomers is difficult, a study of the relationships using synthesized NP isomers is favored.

In this study, various branched isomers of NP and, in addition, the other APs were synthesized and their estrogenic activities were assessed using the yeast two-hybrid system. We investigated the relationships between the structure of the nonyl group of NP isomers and their estrogenic activities. To the best of our knowledge, this research is the first report on the relationships of the pure NP isomers with the estrogenic activities, since 4- $n$-nonylphenol is the only commercially available isomer. We also examined the metabolic effect of the synthesized APs on the estrogenic activity using a liver S9. Finally, we evaluated the estrogenic activities of the synthesized NP isomers in the commercial NP.

\section{MATERIALS AND METHODS}

Reagents — Phenol $(\mathrm{Ph})$ and E2 were purchased from Wako (Osaka, Japan) and 4-tert-pentylphenol (PP 1) and 4-nonylphenol (mixture of NP isomers) from Kishida (Osaka, Japan). The other tested compounds, 4-(pentan-2-yl)phenol (PP 2) 4-(2-methylhexan-2-yl)phenol (HP 1), 4-(3-ethylpentyl)phenol (HP 2), 4-(3,3-dimethylpentan-2-yl)phenol (HP 3), 4-(4,4-dimethylpentan-2-yl)phenol (HP 4), 4-(2methylheptan-2-yl)phenol (OP), 4-(2-methyloctan2-yl)phenol (NP 1), 4-(2,6-dimethylheptan-2- yl)phenol (NP 2), 4-(2,5-dimethylheptan-2-yl)phenol (NP 3), 4-(2,4-dimethylheptan-2-yl)phenol (NP 4), 4-(2,3-dimethylheptan-2-yl)phenol (NP 5), 4-(2,5,5trimethylhexan-2-yl)phenol (NP 6), 4-(2,4,4trimethylhexan-2-yl)phenol (NP 7), 4-(4-ethyl-2methylhexan-2-yl)phenol (NP 8), 4-(nonan-2yl)phenol (NP 9), 4-(nonan-3-yl)phenol (NP 10), 4(nonan-4-yl)phenol (NP 11), 4-(nonan-5-yl)phenol (NP 12), 4-(3-methyloctan-3-yl)phenol (NP 13), 4(4-methyloctan-4-yl)phenol (NP 14), 4-(5-ethylheptan-3-yl)phenol (NP 15), 4-(3-ethylheptyl)phenol (NP 16), 4-(2,2-dimethylheptan-3-yl)phenol (NP 17), 4-(2,6-dimethylheptan-4-yl)phenol (NP 18), 4-(2,2,4,4-tetramethylpentan-3-yl)phenol (NP 19), 4-(3,5,5-trimethylhexan-3-yl)phenol (NP 20), 4(2,2-dimethylheptan-4-yl)phenol (NP 21), 4(3,3,4,4-tetramethylpentan-2-yl)phenol (NP 22), 4-(2-cyclohexylpropan-2-yl)phenol (CNP 1), 4(1-cyclohexylpropyl)phenol (CNP 2), 4-(1cyclohexyl-2-methylpropan-2-yl)phenol (CDP), 4(2-methylundecan-2-yl)phenol (DDP), were synthesized in our laboratory, and the structures were identified by GC-MS (Finnigan MAT GCQ) and ${ }^{1} \mathrm{H}-$ and ${ }^{13} \mathrm{C}-\mathrm{NMR}$ recorded on a JEOL JNM-GSX-400. Greater than a $97 \%$ purity (GC) was obtained for all the synthesized APs.

Measurement of Estrogenic Activity by Yeast Two-Hybrid Assay — The estrogenic activities of the APs were assessed by the yeast two-hybrid system that was first introduced by Nishikawa et $a l .{ }^{20)}$ The estrogenic activity was determined by measuring the $\beta$-galactosidase activity. The yeast two-hybrid assay procedure is as follows: yeast cells (Saccharomyces cerevisiae Y190) introduced with two plasmids (pGBT9-ERLBD and pGAD424TIF2) were preincubated in SD medium at $30^{\circ} \mathrm{C}$. After $18 \mathrm{hr}$, the preincubated yeast cells were diluted ten times with additional SD medium, and $100 \mu \mathrm{l}$ of the solution was transferred to the wells of a 96-well microplate. Dimethyl sulfoxide solutions $(2 \mu \mathrm{l})$ of the test compounds with varying concentrations were added to the culture in the microplate, and the mixtures were incubated. In the case of the metabolic test, the $\mathrm{S} 9 \mathrm{mix}$, which was prepared by mixing an S9 (rat liver induced with phenobarbital and 5,6benzoflavone), water and cofactor $\left(\mathrm{MgCl}_{2} \cdot 6 \mathrm{H}_{2} \mathrm{O}\right.$, $\mathrm{KCl}, \mathrm{G}-6-\mathrm{P}, \mathrm{NADPH}, \mathrm{NADH}, \mathrm{Na}_{2} \mathrm{HPO}_{4}$ and $\mathrm{NaH}_{2} \mathrm{PO}_{4}$ ), was added to the SD medium. After incubation for $18 \mathrm{hr}$ at $30^{\circ} \mathrm{C}$, the absorbances at $620 \mathrm{~nm}$ (optical density at $620 \mathrm{~nm}, \mathrm{OD}_{620}$ ) for the first cell density were read on a microplate reader, and the yeast cells were then digested with $5 \mathrm{mg} / \mathrm{ml}$ 
Zymolyase $20 \mathrm{~T}$ in Z-buffer $(25 \mu \mathrm{l})$ for $30 \mathrm{~min}$ at $30^{\circ} \mathrm{C}$. After the reactions with $0.5 \mathrm{mg} / \mathrm{ml}$ chlorophenol red- $\beta$-D-galactopyranoside (CPRG) in phosphate buffer $(25 \mu \mathrm{l})$ for $1 \mathrm{hr}$ at $30^{\circ} \mathrm{C}, 25 \mu \mathrm{l}$ of $2 \mathrm{M} \mathrm{Na}_{2} \mathrm{CO}_{3}$ solution was added to stop the reactions. The absorbances at $550 \mathrm{~nm}\left(\mathrm{ABS}_{550}\right)$ and $620 \mathrm{~nm}\left(\mathrm{ABS}_{620}\right)$ were read for the measurement of the $\beta$-galactosidase expression and the final cell density, respectively. The $\beta$-galactosidase activity was calculated using the following equation:

$\beta$-galactosidase activity

$$
=\left(\mathrm{ABS}_{550}-\mathrm{ABS}_{620}\right) / \mathrm{OD}_{620} \text {. }
$$

The $10 \%$ relative effective concentration $\left(\mathrm{REC}_{10}\right)$ value is the concentration of the test compound showing $10 \%$ of the highest $\beta$-galactosidase activity of E2. The average value, which was obtained from triplicate measurements of the $\beta$-galactosidase activities, was used for the calculation of the $\mathrm{REC}_{10}$ value. The relative potency (RP) value was estimated by dividing the $\mathrm{REC}_{10}$ value of the commercial NP by that of the test compound.

GC-MS - Analyses of the commercial NP and synthesized NP isomers were performed with a Finnigan MAT GCQ (San Jose, CA, U.S.A.) ion trap mass spectrometer equipped with a Finnigan GC. The column was a Petrocol DH $(100 \mathrm{~m} \times 0.25 \mathrm{~mm}$ $\times 0.5 \mu \mathrm{m}$ film thickness, Supelco, Taufkirchen, Germany). The carrier gas was high purity helium $(99.9999 \%)$ with a constant linear velocity of $20 \mathrm{~cm} / \mathrm{sec}$. The GC oven temperature was programmed as follows: $1 \mathrm{~min}$ at 50 , to $180^{\circ} \mathrm{C}$ at $20^{\circ} \mathrm{C} / \mathrm{min}, 77.5 \mathrm{~min}$ at 180 , to $280^{\circ} \mathrm{C}$ at $20^{\circ} \mathrm{C} / \mathrm{min}$, $10 \mathrm{~min}$ at $280^{\circ} \mathrm{C}$. The ion source, injection and transfer-line temperatures were set at 200,270 and $290^{\circ} \mathrm{C}$, respectively. All injections were performed in the splitless-mode with the split vent closed for $1 \mathrm{~min}$. The mass spectrometer was operated in the electron ionization mode.

\section{RESULTS AND DISCUSSION}

\section{Relationship between Estrogenic Activity and Structure of Nonylphenol Isomers}

The yeast two-hybrid assay was performed at a concentration of less than $1 \times 10^{-5} \mathrm{M}(2.2 \mathrm{mg} / \mathrm{l})$ because the water solubility of the commercial NP (mixture of NP isomers) is about $5.43 \mathrm{mg} / \mathrm{l} .{ }^{21)}$ First of all, the estrogenic activities of $\mathrm{E} 2$ and the commercial NP were measured. The $\mathrm{REC}_{10}$ values of $\mathrm{E} 2$ and the commercial NP were $2.10 \times 10^{-10}$ and $1.13 \times$
$10^{-7} \mathrm{M}$, respectively, and the RP value of E2 was 538. These values were consistent with the previous report. ${ }^{8)}$ Generally, the relative potency of the test compound to E2 was used as the index of the estrogenic activity. In this study, the relative potencies of the APs to the commercial NP were used for the following evaluation of the estrogenic activities, since E2 possessed much a higher estrogenic activity than the commercial NP.

Because the NP isomers, which possess the different branches in the nonyl group, have varying lengths of the main alkyl chain, the information on the effect of the length of the main alkyl chain for the estrogenic activity was essential. Evaluating the effect of the length of the alkyl group using the recombinant yeast screen, Routledge and Sumpter ${ }^{13)}$ reported that the octylphenol, which possesses the $\alpha, \alpha$-dimethyl branch in the octyl group, showed the maximum estrogenic activity. In the case of the yeast two-hybrid assay, the estrogenic activities of $\mathrm{Ph}, \mathrm{PP}$ 1, HP 1, OP, NP 1 and DDP were studied to confirm the effect of the length of the main alkyl chain (Table 1). Ph and DDP did not stimulate the $\beta$-galactosidase activity at all, while HP $\mathbf{1}$ showed the highest estrogenic activity among these compounds. The appropriate length of the main alkyl chain was suggested to be essential for possessing the estrogenic activity. Comparing these compounds with the commercial NP, NP 1 showed a 14 times lower estrogenic activity, in addition, even HP 1 presented a seven times lower estrogenic activity. This might suggest that any other factor was important for possessing the "high" estrogenic activity.

For the investigation of the important factor for the high estrogenic activity, the estrogenic activities of the $\alpha, \alpha$-dimethyl NPs having several branches on the main alkyl chain were measured. The results of NPs 2-5 having the methyl branch on the $\varepsilon^{-}, \delta-, \gamma$ and $\beta$-carbons, respectively, NPs 6 and 7 having the dimethyl branch on the $\gamma$ and $\delta$-carbons, respectively, and NP 8 having the ethyl branch on the $\gamma$-carbon are shown in Table 2. By changing the branch except for the $\alpha$-carbon, the estrogenic activities were significantly varied. Comparing NPs 2-5, NPs 3-5 showed higher estrogenic activities than NP 2. Moreover, about a five times higher activity of NP 7 was obtained compared to that of NP 6 . This might suggest that the branching position in the nonyl group was important. Comparing the NP isomers having the branch on the $\gamma$-carbon, NPs $\mathbf{7}$ and $\mathbf{8}$, which are bulkier on the $\gamma$-carbon than NP 4, had higher estrogenic activities. A similar tendency was monitored 
Table 1. REC 10 and RP Values of Ph, PP 1, HP 1, OP, NP 1 and DDP in Yeast Two-Hybrid Assay

\begin{tabular}{|c|c|c|c|}
\hline & compound & $\mathrm{REC}_{10}{ }^{a)}(\mathrm{M})$ & $\overline{\mathrm{RP}^{b)}}$ \\
\hline $\mathrm{Ph}$ & & Nonactive $^{c)}$ & - \\
\hline PP 1 & & $3.08 \times 10^{-6}$ & 0.037 \\
\hline HP 1 & & $8.26 \times 10^{-7}$ & 0.14 \\
\hline OP & & $1.39 \times 10^{-6}$ & 0.081 \\
\hline NP 1 & & $1.61 \times 10^{-6}$ & 0.070 \\
\hline DDP & & Nonactive $^{c)}$ & - \\
\hline
\end{tabular}

Table 2. REC $_{10}$ and RP Values of NPs 2-8 in Yeast Two-Hybrid Assay

(2)


Table 3. REC $_{10}$ and RP Values of NPs $\mathbf{1}$ and 9-14 in Yeast Two-Hybrid Assay

(1)

for the evaluation of the $\delta$-branch; NP 6 having the dimethyl branch on the $\delta$-carbon showed a higher estrogenic activity than NP 3. These results indicated that the bulkiness around the $\gamma$-and $\delta$-carbons was important for the high estrogenic activity. It was reported that the C- and D-rings of E2 play an important role in stabilizing the ligand-receptor complex by the hydrophobic interactions. ${ }^{22,23)}$ In the case of NPs 6-8, the dimethyl or ethyl branch on the $\gamma$ or $\delta$ carbon might play a role in the hydrophobic $\mathrm{C}$ and/ or $\mathrm{D}$ ring of $\mathrm{E} 2$.

The tert-branch on the $\alpha$-carbon was reported to be an important feature for the estrogenic activity. ${ }^{13)}$ Therefore, the estrogenic activities of the NP isomers having various branches on the $\alpha$-carbon (NPs 1 and 9-14) were examined. As can be seen in Table 3, no dramatic difference in the estrogenic activities between NPs $\mathbf{1}$ and 9-14 was observed (RP $=0.07-0.14)$, and these NP isomers similarly showed more than five times lower estrogenic activities than the commercial NP. These results might indicate that the branch on the $\alpha$-carbon slightly affected the estrogenic activity, in addition, the NP isomers having the branch only on the $\alpha$-carbon showed a relatively low estrogenic activity. We also evaluated the effect of the $\alpha$-branch using HP 2 and NPs 8 and 15 which have different branches on the $\alpha$-carbon with the same length of the main alkyl chain and ethyl branch on the $\gamma$-carbon. As shown in Table 4, similar estrogenic activities by HP 2 and NPs 8 and 15 were obtained. This result also indicated the slight effect of the branch on the $\alpha$-carbon for the estrogenic activity. The lower estrogenic activity of NP 16 than that of HP 2 might be due to the long main alkyl chain. The effect of the $\alpha$-branch in this study was somewhat different from the research by Routledge and Sumpter. ${ }^{13)}$ This might be due to the difference in the tested alkylphenols and/or bioassay method. Hashimoto et al. $^{24)}$ reported that the difference in the bioassay method affected the sensitivity of the estrogenic activity.

Next, the relationship between the estrogenic activity and the bulkiness in the nonyl group was investigated using NPs 12 and 17-19. NPs 12 and 17-19 have di-n-butyl, $n$-butyl-tert-butyl, di-isobutyl and di-tert-butyl groups on the $\alpha$-carbon, respectively. As shown in Table 5, the NP isomers, which have more branches in the nonyl group, showed higher estrogenic activities. The importance of the bulkiness in the nonyl group was confirmed. 
Table 4. REC 10 and RP Values of HP 2 an NPs 8, 15 and 16 in Yeast Two- Hybrid Assay

(15 2

Table 5. REC 10 and RP Values of NPs 12 and 17-21 in Yeast Two-Hybrid Assay

(12 21

The bulky NP isomers, NPs 20 and 21, were also synthesized and their estrogenic activities were assessed. To our expectation, NPs 20 and 21 showed higher estrogenic activities compared with NPs 13 and 11, respectively. The similar RP values of NPs 20 and 21 were good agreement with the result in the evaluation of the effect of the branch on the $\alpha$ carbon.

From the results of Tables $1-5$, the importance of the appropriate length of the main alkyl chain and the bulkiness on the nonyl group (especially around the $\gamma$-carbons) for possessing the high estrogenic activity was indicated. The branching on the $\alpha$-carbon slightly affected the estrogenic activity. Moreover, the importance of the bulkiness on the $\beta$-carbon for the high estrogenic activity might be suggested due to the highest estrogenic activity of NP 19 among the tested APs in Tables 1-5. In view of 
Table 6. REC $_{10}$ and RP Values of NP 22, PP 2 and HPs 3 and 4 in Yeast Two-Hybrid Assay

\begin{tabular}{|c|c|c|c|}
\hline & compound & $\operatorname{REC}_{10}(\mathrm{M})$ & $\mathrm{RP}$ \\
\hline NP 22 & & $7.37 \times 10^{-10}$ & 153 \\
\hline PP 2 & & $1.13 \times 10^{-6}$ & 0.083 \\
\hline HP 3 & & $1.41 \times 10^{-8}$ & 8.0 \\
\hline HP 4 & & $6.18 \times 10^{-8}$ & 1.8 \\
\hline
\end{tabular}

Table 7. $\mathrm{REC}_{10}$ and RP Values of CNPs 1 and $\mathbf{2}$ and CDP in Yeast Two-Hybrid Assay

\begin{tabular}{|c|c|c|c|}
\hline & compound & $\mathrm{REC}_{10}(\mathrm{M})$ & $\mathrm{RP}$ \\
\hline CNP 1 & & $8.06 \times 10^{-9}$ & 14 \\
\hline CNP 2 & & $3.02 \times 10^{-9}$ & 37 \\
\hline $\mathrm{CDP}$ & & $1.97 \times 10^{-7}$ & 0.57 \\
\hline
\end{tabular}

this suggestion, NP 22, which was expected to possess the high estrogenic activity, was synthesized and evaluated for its estrogenic activity in the range from $1.00 \times 10^{-5}$ to $4.77 \times 10^{-12} \mathrm{M}$. To our surprise, NP 22 showed a 153 times higher estrogenic activity than the commercial NP (Table 6). As described above, we proposed that the branch on the $\alpha$-carbon might be slightly related to the estrogenic activity. It was assumed that the dimethyl branches on the $\beta$ - and $\gamma$ carbons induced NP 22 to have an extremely high activity. To elucidate the structure attributed to the extremely high activity, PP 2, HPs $\mathbf{3}$ and $\mathbf{4}$ were synthesized and their estrogenic activities were measured. As shown in Table 6, HP 3, which has the dimethyl branch on the $\beta$-carbon, showed a higher estrogenic activity than PP 2 and HP 4. This might suggest that the bulkiness on the $\beta$-carbon was significant for the estrogenic activity. Moreover, similar to the high estrogenic activity of NPs $\mathbf{7}, \mathbf{8}, \mathbf{1 5}$, 20, and 21, the importance of the bulkiness on the $\gamma$ carbon was indicated from the relatively high activity of HP 4. Comparing the $\mathrm{REC}_{10}$ values between
PP 2 and HP 3 and between HP 4 and NP 22, the $\beta$ dimethyl branch induced about a 90 times higher estrogenic activity, while comparing the $\mathrm{REC}_{10}$ values between PP $\mathbf{2}$ and HP $\mathbf{4}$ and between HP $\mathbf{3}$ and NP 22, the $\gamma$-dimethyl branch induced about a 20 times higher activity. NP 7 having the dimethyl branch on the $\gamma$-carbon similarly showed about a 20 times higher estrogenic activity than HP $\mathbf{1}$. The $\mathrm{X}$-ray study of the ligand binding domain with E2 indicated the presence of a large unoccupied space around the $\mathrm{C}$-ring. On the other hand, the $\mathrm{A}$ - and $\mathrm{D}$ ring ends of E2 were held tightly by ER $\alpha{ }^{6}{ }^{6}$ It might be suggested that the projected dimethyl branch on the $\beta$-carbon penetrated into the free space in the ligand binding domain. Though such molecular docking and modeling study on the recognition with $\mathrm{ER} \alpha$ may be required to clearly illuminate the extremely high estrogenic activity of NP 22, we concluded that the dimethyl branch on the $\beta$-carbon significantly enhanced the estrogenic activity.

The estrogenic activities of CNPs $\mathbf{1}$ and $\mathbf{2}$ having the cyclic structure were evaluated. As shown in 


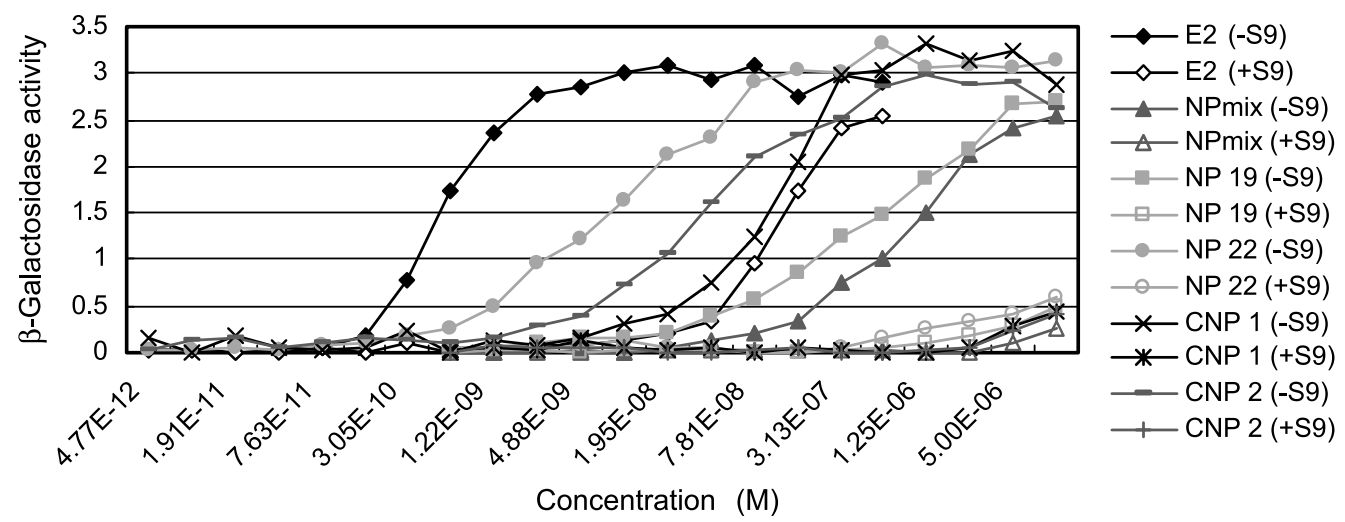

Fig. 1. Dose-Response Curves of Estrogenic Activities of E2, NPmix (commercial NP), NPs 19 and 22 and CNPs 1 and 2 in Yeast TwoHybrid Assay with S9 (+S9) and without S9 (-S9)

Average values from the triplicate analyses were plotted.

Table 7, CNPs 1 and 2 gave high estrogenic activities. CNPs $\mathbf{1}$ and $\mathbf{2}$ have a cyclohexyl group on the $\alpha$-carbon, in other words, have a branch on the $\beta$ carbon. To evaluate the effect of the branch on the $\beta$-carbon, CDP having a cyclohexyl group on the $\beta$ carbon (branch on the $\gamma$-carbon) was synthesized and its estrogenic activity was assessed. According to our expectation, CDP showed more than a 20 times lower estrogenic activity than CNP 1 (Table 7). Although the longer main alkyl chain of CDP might also be the possible reason for the lower estrogenic activity of CDP than that of CNP 1, the high estrogenic activities of CNPs $\mathbf{1}$ and $\mathbf{2}$ were suggested to be due to the bulkiness around the $\beta$-carbon.

\section{Metabolic Effect of Synthesized NP Isomers on Estrogenic Activity}

The effect of the metabolism by a rat liver $\mathrm{S} 9$ on the estrogenic activities of the E2, commercial NP (NPmix), NPs 1, 4, 8, 19, and 22 and CNPs 1 and 2 were examined. NPs $\mathbf{1}, \mathbf{4}$, and $\mathbf{8}$ showed no estrogenic activities with $\mathrm{S} 9 \mathrm{mix}$ at a concentration of less than $1 \times 10^{-5} \mathrm{M}$. The $\beta$-galactosidase responses of the other test compounds with S9 mix and without S9 mix are given in Fig. 1. NPs 19 and $\mathbf{2 2}$ and CNPs 1 and 2, which showed high estrogenic activities in the absence of the $\mathrm{S} 9 \mathrm{mix}$, produced the decreased estrogenic activities with the S9 mix $\left(\mathrm{REC}_{10}\right.$ values $=4.25 \times 10^{-6}, 1.44 \times 10^{-6}, 8.32 \times 10^{-6}$ and $7.65 \times 10^{-6} \mathrm{M}$, respectively). The commercial NP showed less than $10 \%$ of the highest estrogenic activity of E2. These metabolic effects might be due to the conversion of APs to their oxidative metabolites. The difference in the structure of the alkyl group only slightly affected the estrogenic activity with a rat liver S9.

\section{Estrogenic Activity of Synthesized NP Isomers in Commercial NP}

The commercial NP and synthesized NP isomers were analyzed by GC-MS for the identification of the NP isomers in the commercial NP. From the retention times and mass spectra, NPs 1-6, 8, 13, and 14 were suggested to be present in the commercial NP. The NP 22 having extremely high estrogenic activity was not included in the commercial NP. The presence of these isomers were good agreement with previous reports. ${ }^{17,18,25,26)}$ These NP isomers showed lower estrogenic activities than the commercial NP. The other NP isomers in the commercial NP were suggested to possess much higher estrogenic activities compared with the NP isomers synthesized in this study. Very recently, Kim et al. ${ }^{26)}$ fractionalized 4-(3-ethyl-2-methylhexan-2-yl)phenol from the commercial NP, and its estrogenic activity assessed by the recombinant yeast screen assay showed the highest estrogenic activity among the isomeric fractions. Since the isomer has the ethyl branch on the $\beta$-carbon, the high estrogenic activity of the commercial NP may be ascribed to 4-(3-ethyl-2methylhexan-2-yl)phenol.

In conclusion, we reported that the difference in the nonyl group dramatically affected the estrogenic activity of the NP isomer. To certify the reliability of the estrogenic activities of the NP isomers, the measurement by in vivo assay is suggested to be required. ${ }^{27)}$ The results from the yeast two-hybrid assay presented that the bulkiness in the nonyl group was suggested to be an important feature for the high estrogenic activity. Moreover, the position of the 
bulky site in the nonyl group, especially the bulkiness on the $\beta$-carbon, was suggested to be the most important factor for the estrogenic activity.

\section{REFERENCES}

1) Colborn, T., Dumanoski, D. and Myers, J. P. (1996) Our Stolen Future, A Dutton Book Press, New York.

2) Shelby, M. D., Newbold, R. R., Tully, D. B., Chae, K. and Davis, V. L. (1996) Assessing environmental chemicals for estrogenicity using a combination of in vitro and in vivo assays. Environ. Health Perspect., 104, 1296-1300.

3) Tapiero, H., Nguyen, B. G. and Tew, K. D. (2002) Estrogens and environmental estrogens. Biomed. Pharmacother., 56, 36-44.

4) White, R., Jobling, S., Hoare, S. A., Sumpter, J. P. and Parker, M. G. (1994) Environmentally persistent alkylphenolic compounds are estrogenic. Endocrinology, 135, 175-182.

5) Routledge, E. J. and Sumpter, J. P. (1996) Estrogenic activity of surfactants and some of their degradation products assessed using a recombinant yeast screen. Environ. Toxicol. Chem., 15, 241-248.

6) Brzozowski, A. M., Pike, A. C. W., Dauter, Z., Hubbard, R. E., Bonn, T., Engström, O., Öhman, L., Greene, G. L., Gustafsson, J. A. and Carlquist, M. (1997) Molecular basis of agonism and antagonism in the oestrogen receptor. Nature (London), 389, 753-758.

7) Pedersen, S. N., Christiansen, L. B., Pedersen, K. L., Korsgaard, B. and Bjerregaard, P. (1999) In vivo estrogenic activity of branched and linear alkylphenols in rainbow trout (Oncorhynchus mykiss). Sci. Total Environ., 233, 89-96.

8) Nishihara, T., Nishikawa, J., Kanayama, T., Dakeyama, F., Saito, K., Imagawa, M., Takatori, S., Kitagawa, Y., Hori, S. and Utsumi, H. (2000) Estrogenic activities of 517 chemicals by yeast two-hybrid assay. J. Health Sci., 46, 282-298.

9) Schmieder, P. K., Aptula, A. O., Routledge, E. J., Sumpter, J. P. and Mekenyan, O. G. (2000) Estrogenicity of alkylphenolic compounds: a 3-D structure-activity evaluation of gene activation. Environ. Toxicol. Chem., 19, 1727-1740.

10) Yamakoshi, Y., Otani, Y., Fujii, S. and Endo, Y. (2000) Dependence of estrogenic activity on the shape of the 4-alkyl substituent in simple phenols. Biol. Pharm. Bull., 23, 259-261.

11) Fang, H., Tong, W., Shi, L. M., Blair, R., Perkins, R., Branham, W., Hass, B. S., Xie, Q., Dial, S. L., Moland, C. L. and Sheehan, D. M. (2001) Struc- ture-active relationships for a large diverse set of natural, synthetic, and environmental estrogens. Chem. Res. Toxicol., 14, 280-294.

12) Tabira, Y., Nakai, M., Asai, D., Yakabe, Y., Tahara, Y., Shinmyozu, T., Noguchi, M., Takatsuki, M. and Shimohigashi, Y. (1999) Structural requirements of para-alkylphenols to bind to estrogen receptor. Eur. J. Biochem., 262, 240-245.

13) Routledge, E. J. and Sumpter, J. P. (1997) Structural features of alkylphenolic chemicals associated with estrogenic activity. J. Biol. Chem., 272, 32803288.

14) Schultz, T. W., Sinks, G. D. and Cronin, M. T. D. (2000) Effect of substituent size and dimensionality on potency of phenolic xenoestrogens evaluated with a recombinant yeast assay. Environ. Toxicol. Chem., 19, 2637-2642.

15) Kojima, M., Tsunoi, S. and Tanaka, M. (2004) High performance solid-phase analytical derivatization of phenols for gas chromatography-mass spectrometry. J. Chromatogr. A, 1042, 1-7.

16) Ferrara, F., Fabietti, F., Delise, M. and Funari, E. (2005) Alkylphenols and alkylphenol ethoxylates contamination of crustaceans and fishes from the Adriatic sea (Italy). Chemosphere, 59, 1145-1150.

17) Thiele, B., Heinke, V., Kleist, E. and Günther, K. (2004) Contribution to the structural elucidation of 10 isomers of technical p-nonylphenol. Environ. Sci. Technol., 38, 3405-3411.

18) Kim, Y. S., Katase, T., Sekine, S., Inoue, T., Makino, M., Uchiyama, T., Fujimoto, Y. and Yamashita, N. (2004) Variation in estrogenic activity among fractions of a commercial nonylphenol by high performance liquid chromatography. Chemosphere, 54, 1127-1134.

19) Yamashita, N., Kannan, K., Hashimoto, S., Miyazaki, A. and Gisey, J. P. (1999) Estrogenic potency of individual nonylphenol congener isolated from technical mixtures. Organohalogen Compd., 42, 121-123.

20) Nishikawa, J., Saito, K., Goto, J., Dakeyama, F., Matsuo, M. and Nishihara, T. (1999) New screening methods for chemicals with hormonal activities using interaction of nuclear hormone receptor with coactivator. Toxicol. Appl. Pharmacol., 154, 76-83.

21) Ahel, M. and Giger, W. (1993) Aqueous solubility of alkylphenols and alkylphenol ethoxylates. Chemosphere, 26, 1461-1470.

22) Elsby, R., Ashby, J., Sumpter, J. P., Brooks, A. N., Pennie, W. D., Maggs, J. L., Lefevre, P. A., Odum, J., Beresford, N., Paton, D. and Park, K. (2000) Obstacles to the prediction of estrogenicity from chemical structure: assay-metabolic transformation and the apparent promiscuous nature of the estrogen recep- 
tor. Biochem. Pharmacol., 60, 1519-1530.

23) Endo, Y., Iijima, T., Yamakoshi, Y., Fukasawa, H., Miyaura, C., Inada, M., Kubo, A. and Itai, A. (2001) Potent estrogen agonists based on carborane as a hydrophobic skeletal structure a new medicinal application of boron clusters. Chem. Biol., 8, 341-355.

24) Hashimoto, Y., Moriguchi, Y., Oshima, H., Kawaguchi, M., Miyazaki, K. and Nakamura, M. (2001) Measurement of estrogenic activity of chemicals for the development of new dental polymers. Toxicol. In Vitro, 15, 421-425.

25) Ruß, A. S., Vinken, R., Schuphan, I. and Schmidt, B. (2005) Synthesis of branched para-nonylphenol isomers: Occurrence and quantification in two commercial mixtures. Chemosphere, 60, 1624-1635.

26) Kim, Y. S., Katase, T., Horii, Y., Yamashita, N., Makino, M., Uchiyama, T., Fujimoto, Y. and Inoue, T. (2005) Estrogen equivalent concentration of individual isomer-specific 4-nonylphenol in Ariake sea water, Japan. Mar. Pollut. Bull., 51, 850-856.

27) Belt, K. V., Berckmans, P., Vangenechten, C., Verheyen, R. and Witters, H. (2004) Comparative study on the in vitro/in vivo estrogenic potencies of $17 \beta$-estradiol, estrone, $17 \alpha$-ethynylestradiol and nonylphenol. Aquat. Toxicol., 66, 183-195. 